\title{
Coding without Fine Structure
}

\author{
Sy D. Friedman!
}

M.I.T.

In this paper we prove Jensen's Coding Theorem, assuming $\sim 0^{\#}$, via a proof that makes no use of the fine structure theory. We do need to quote Jensen's Covering Theorem, whose proof uses fine-structural ideas, but make no direct use of these ideas. The key to our proof is the use of "coding delays."

Coding Theorem (Jensen) Suppose $\langle M, A\rangle$ is a model of $Z F C+O^{\#}$ does not exist. Then there is an $\langle M, A\rangle$-definable class forcing $P$ such that if $G \subseteq P$ is $P$-generic over $\langle M, A\rangle$ :

(a) $\langle M[G], A, G\rangle \models Z F C$. $R$.

(b) $M[G] \models V=L[R], R \subseteq \omega$ and $\langle M[G], A, G\rangle \models A, G$ are definable from the parameter

In the above statement when we say " $\langle M, A\rangle \models Z F C$ " we mean that $M \models Z F C$ and in addition $M$ satisfies replacement for formulas that mention $A$ as a predicate. And " $P$ generic over $\langle M, A\rangle$ " means that all $\langle M, A\rangle$-definable dense classes are met.

The consequence of $\sim O^{\#}$ that we need follows directly from the Covering Theorem.

Covering Theorem (Jensen) Assume $\sim O^{\#}$. If $X$ is an uncountable set of ordinals then there is a constructible $Y \supseteq X$, card $Y=\operatorname{card} X$.

Lemma 1 (Jensen) Assume $\sim O^{\#}$. If $j: L_{\alpha} \longrightarrow L_{\beta}$ is $\Sigma_{1}$-elementary, $\alpha \geq \omega_{2}$ and $\kappa=\operatorname{crit}(j)$ then $\alpha<\left(\kappa^{+}\right)^{L}$.

Proof Of course $\operatorname{crit}(j)$ denotes the least ordinal $\kappa$ such that $j(\kappa) \neq \kappa$, which we assume to exist. Now let $U=\left\{X \subseteq \kappa \mid X \in L_{\alpha}, \kappa \in j(X)\right\}$. If $\alpha \geq\left(\kappa^{+}\right)^{L}$ then $U$ is an ultrafilter on all constructible subsets of $\kappa$ and we can form $U l t(L, U)=$ ultrapower of $L$ by $U$ (using constructible functions to form the ultrapower). If this is well-founded then we get a nontrivial elementary embedding $L \longrightarrow L$, which gives $O^{\#}$ by a theorem of Kunen.

Now we know that $\operatorname{Ult}\left(L_{\alpha}, U\right)$ is well-founded since it embeds into $L_{\beta}$ (using: $k([f])=$ $j(f)(\kappa))$. And by a Lowenheim-Skolem argument, if $U l t(L, U)$ were ill-founded then so would be $U l t\left(L_{\kappa^{+}}, U\right), \kappa^{+}=$the real $\kappa^{+}$. So we may assume that $\kappa \geq \omega_{2}$ as otherwise $\kappa^{+} \leq \omega_{2} \leq \alpha$ and the facts above would imply that $U l t(L, U)$ were well-founded.

Using the Covering Theorem and the fact that $\kappa \geq \omega_{2}$ we show that if $\left\langle X_{n} \mid n \in \omega\right\rangle$ belong to $U$ then $\bigcap_{n} X_{n} \neq \phi$ ( $U$ is "countably complete"), a fact that immediately yields the well-foundedness of $U l t(L, U)$.

Apply Covering to get $F \in L$ of cardinality $\omega_{1}$ such that $X_{n} \in F$ for each $n$. As $\kappa \geq$ $\omega_{2}, F$ has $L$-cardinality $<\kappa$ and also we may assume that $F$ is a subset of $P(\kappa) \cap L$. So $F \in L_{\left(\kappa^{+}\right)^{L}} \subseteq L_{\alpha}$ and there is a bijection $h: F \longrightarrow \gamma, \gamma<\kappa, h \in L_{\alpha}$. Let $F^{*}=\{X \in F \mid \kappa \in$ $\mathrm{J}(X)\}$; then $F^{*} \in L_{\alpha}$ since $h\left[F^{*}\right]=\{j(h)(Y) \mid Y \in j(F), \kappa \in Y\}$ belongs to $L_{\beta}$ and hence to $L_{\kappa} \subseteq L_{\alpha}$. So $\cap F^{*} \neq \phi$ since $j\left(\cap F^{*}\right)=\cap j\left[F^{*}\right]$ contains $\kappa$ and $j$ is $\Sigma_{1}$-elementary. As $\left\{X_{n} \mid n \in \omega\right\} \subseteq F^{*}$ we get $\bigcap_{n} X_{n} \neq \phi$, as desired. $\dashv$

\footnotetext{
${ }^{1}$ Research supported by NSF Contract \# 9205530.
} 
Next we show that to prove the Coding Theorem we may assume that the GCH holds in $M$, and that instead of coding into a real, it is enough to code into a "reshaped" subset of $\omega_{1}$.

Lemma 2 (Folklore) If $\langle M, A\rangle$ is a model of ZFC then there is an $\langle M, A\rangle$-definable forcing $P^{*}$ such that if $G^{*}$ is $P^{*}$-generic over $\langle M, A\rangle$ then for some $B \subseteq \operatorname{ORD}(M), B$ is definable over $\left\langle M\left[G^{*}\right], A, G^{*}\right\rangle$ and this model satisfies $Z F C+G C H+V=L[B]+A, G^{*}$ are definable relative to $B$. And if $M$ satisfies $\sim O^{\#}$ then so does $M\left[G^{*}\right]$.

Proof First, by forcing with conditions $p: \alpha \longrightarrow 2, \alpha \in O R D$, ordered by $p \leq q$ iff $p$ extends $q$ we can obtain $B$ as above, except for the GCH. This is beause if $G_{0}^{*}$ is generic for this forcing and $B_{0}=\left\{\beta \mid p(\beta)=1\right.$ for some $\left.p \in G_{0}^{*}\right\}$ then $M\left[G_{0}^{*}\right] \models V=L\left[B_{0}\right]$ and using $B_{0}$ we can identify $A$ with a class of ordinals $B_{1}$; let $B=$ the join of $B_{0}, B_{1}$.

Second, we force over $\langle L[B], B\rangle$ to obtain the GCH. As usual, $\sqsupset_{\alpha}$ is defined (in $L[B]$ ) by: $\sqsupset_{0}=\omega, \sqsupset_{\alpha+1}=2^{\sqsupset_{\alpha}}$ and $\sqsupset_{\lambda}=\cup\left\{\sqsupset_{\alpha} \mid \alpha<\lambda\right\}$ for limit $\lambda$. For any $\alpha P(\alpha)$ is the forcing whose conditions are $p: \beta \longrightarrow 2^{\sqsupset_{\alpha}}, \beta<\sqsupset_{\alpha}^{+}$, ordered by $p \leq q$ iff $p$ extends $q$. We take $P$ to be the "Easton product" of the $P(\alpha)$ 's: a condition in $P$ is $p: \alpha(p) \longrightarrow L[B], p \in L[B]$ such that $p(\alpha) \in P(\alpha)$ for each $\alpha<\alpha(p)$ and such that $\{\beta<\alpha \mid p(\beta) \neq \phi\}$ is bounded in $\alpha$ for inaccessible $\alpha \leq \alpha(p)$. For any $\alpha P$ factors as $P(>\alpha) \times P(\leq \alpha)$ where $P(>\alpha)$ is $\sqsupset_{\alpha+1}^{+}$-closed and $P(\leq \alpha)$ has cardinality $\leq \sqsupset_{\alpha+1}$. It follows that ZFC is preserved, the infinite successor cardinals of the generic extension are the $\sqsupset_{\alpha}^{+}$of $L[B]$ and that the GCH holds in the generic extension. And if $L[B]$ satisfies $\sim O^{\#}$ then so does the $P$-generic extension, since for singular strong limit cardinals $\kappa$ of $L[B], \kappa^{+}$of $L[B]=\kappa^{+}$of $L$ and $\kappa^{+}$ of $L[B]=\kappa^{+}$of the $P$-generic extension.

Let $P^{*}$ be the product of the two forcings described above. $\dashv$

Definition $b \subseteq \omega_{1}$ is reshaped if $\xi<\omega_{1} \longrightarrow \xi$ is countable in $L[b \cap \xi]$.

Lemma 3 (Jensen-Solovay [68]) Suppose $M \models Z F C+V=L[b]$ where $b$ is a reshaped subset of $\omega_{1}$. Then there is a CCC forcing $P$ such that if $G$ is $P$-generic over $M$ then $M[G] \models V=L[R]$ where $R \subseteq \omega$.

Proof Using the fact that $b$ is reshaped we may choose $\left\langle R_{\xi}^{\prime} \mid \xi<\omega_{1}\right\rangle$ so that for each $\xi<\omega_{1}$, $R_{\xi}^{\prime}$ is the least real in $L[b \cap \xi]$ distinct from each $R_{\xi^{\prime}}^{\prime}, \xi^{\prime}<\xi$. Let $R_{\xi}=\{n<\omega \mid n$ codes a finite initial segment of the characteristic function of $\left.R_{\xi}^{\prime}\right\}$. Then $\xi_{0} \neq \xi_{1} \longrightarrow R_{\xi_{0}} \cap R_{\xi_{1}}$ is finite.

A condition in $P$ is $p=\left(s(p), s^{*}(p)\right)$ where $s(p)$ is a finite subset of $\omega$ and $s^{*}(p)$ is a finite subset of $b$. Extension is defined by: $p \leq q$ iff $s(p)$ end extends $s(q), s^{*}(p) \supseteq s^{*}(q)$ and $\xi \in s^{*}(q) \longrightarrow s(p)-s(q)$ is disjoint from $R_{\xi}$. This is ccc and if $G$ is $P$-generic, $R=\cup\{s(p) \mid p \in G\}$ then $\xi \in b$ iff $R \cap R_{\xi}$ is finite. So inductively we can recover $b \cap \xi, R_{\xi}$ in $L[R]$. And $p \in G$ iff $s(p)$ is an initial segment of $R, \xi \in s^{*}(p) \longrightarrow R_{\xi} \cap R \subseteq s(p)$. So $M[G] \models V=L[b, G]=L[R] . \dashv$

Thus the Coding Theorem with $\sim O^{\#}$ reduces to:

Theorem 4 Suppose that $A \subseteq \mathrm{ORD}$ and $\langle L[A], A\rangle$ is a model of $\mathrm{ZFC}+\mathrm{GCH}+\sim$ $O \#$. Then there is an $\langle L[A], A\rangle$-definable class forcing $P$ such that if $G$ is $P$-generic over $\langle L[A], A\rangle$ : 
(a) $\langle L[A, G], A, G\rangle$ is a model of ZFC.

(b) $L[A], L[A, G]$ have the same cofinalities.

(c) $L[A, G]=L[X]$ where $X$ is a reshaped subset of $\omega_{1}$ and $A, G$ are definable over $L[X]$ with parameter $X$.

It is useful to make the following harmless assumption about $A$ : if $H_{\alpha}, \alpha$ an infinite $L[A]$-cardinal, denotes $\{X \in L[A] \mid$ transitive closure $(X)$ has $L[A]$-cardinality $<\alpha\}$ then $H_{\alpha}=L_{\alpha}[A]$. This is easily arranged using the GCH in $L[A]$.

\section{Definition of the Forcing $\mathbf{P}$}

Let Card $=$ all infinite cardinals, Card $^{+}=\left\{\alpha^{+} \mid \alpha \in\right.$ Card $\}$ and Card $^{\prime}=$ all uncountable limit cardinals. Of course these definitions are made in $V=L[A]$.

Definition (Strings) Let $\alpha \in$ Card. $S_{\alpha}$ consists fo all $s:[\alpha,|s|) \longrightarrow 2, \alpha \leq|s|<\alpha^{+}$ such that $|s|$ is a multiple of $\alpha$ and for all $\eta \leq|s|, L_{\delta}[A \cap \alpha, s\lceil\eta] \models \operatorname{card}(\eta) \leq \alpha$ for some $\delta<\left(\eta^{+}\right)^{L} \cup \omega_{2}$.

Thus for $\alpha \geq \omega_{2}$ we insist that $s$ is "quickly reshaped" in that $\eta \leq|s|$ is collapsed relative to $A \cap \alpha, s \uparrow \eta$ before $\left(\eta^{+}\right)^{L}$. This will enable us to establish cofinality-preservation, using Lemma 1. Note that we allow $|s|=\alpha$, in which case $s=\phi_{\alpha}$, the "empty string at $\alpha . "$ Also for $s, t \in S_{\alpha}$ write $s \leq t$ for $s \subseteq t$ and $s<t$ for $s \leq t, s \neq t$.

Definition (Coding Structures) For $s \in S_{\alpha}$ defome $\mu^{<s}, \mu^{s}$ inductively by: $\mu^{<\phi_{\alpha}}=$ $\alpha, \mu^{<s}=\cup\left\{\mu^{t} \mid t<s\right\}$ for $s \neq \phi_{\alpha}$ and $\mu^{s}=$ least $\mu>\mu^{<s}$ such that $\mu^{\prime} \mu=\mu$ for $\mu^{\prime}<\mu$ and $L_{\mu}[A \cap \alpha, s] \models " s \in S_{\alpha}$ ". And $\mathcal{A}^{\}}=\mathcal{L}_{\mu^{\jmath}}\left[\mathcal{A} \cap \alpha, \int\right], \mathcal{A}^{<\mathcal{S}}=\left\langle\mathcal{L}_{\mu^{<j}}[\mathcal{A} \cap \alpha, \hat{\jmath}], \mathcal{A} \cap \alpha, \hat{\jmath}\right\rangle$ where $\hat{s}=\left\{\mu^{<t}+\delta \mid t<s, \delta<\alpha, s(|t|+\delta)=1\right\}$.

Thus by definition there is $\delta<\mu^{s}$ such that $L_{\delta}[A \cap \alpha, s] \models \operatorname{card}(|s|) \leq \alpha$ and $L_{\mu^{s}} \models$ $\operatorname{card}(\delta) \leq|s|$, when $\alpha \geq \omega_{2}$. For $|s|=\eta+\alpha, \eta$ a multiple of $\alpha, \mathcal{A}^{<\int}$ has universe $\mathcal{A}^{\int\lceil\eta}$ and

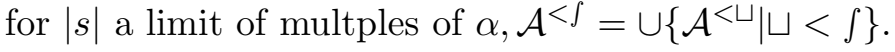

Definition (Coding Apparatus) For $\omega \neq \alpha \in$ Card, $s \in S_{\alpha}, i<\alpha$ let $H^{s}(i)=\Sigma_{1}$ Hull of $i \cup\{A \cap \alpha, s\}$ in $\mathcal{A}^{\int}$ and $f^{s}(i)=$ ordertype $\left(H^{s}(i) \cap \mathrm{ORD}\right)$. For $\alpha \in \operatorname{Card}^{+}, b^{s}=\operatorname{Range}\left(f^{s} \uparrow\right.$ $\left.B^{s}\right)$ where $B^{s}=\left\{i<\alpha \mid i=H^{s}(i) \cap \alpha\right\}$. Also for $\eta<|s|, \eta=|t|+\delta, \delta<\alpha, t<s$ we define $b^{s \mid \eta}=\left\{\gamma+\delta \mid \gamma \in b^{t}\right\}$.

Definition (A Partition of the Ordinals) Let $B, C, D, E$ denote the classes of ordinals congruent to $0,1,2,3 \bmod 4$, respectively. Also for any ordinal $\alpha$ and $X=B, C, D$ or $E$ we write $\alpha^{X}$ for the $\alpha^{\text {th }}$ element of $X$.

Definition (The Successor Coding) Suppose $\alpha \in$ Card, $s \in S_{\alpha^{+}}$. A condition in $R^{s}$ is a pair $\left(t, t^{*}\right)$ where $t \in S_{\alpha}, t^{*} \subseteq\left\{b^{s \mid \eta}|\alpha \leq \eta<| s \mid\right\}$, $\operatorname{card}\left(t^{*}\right) \leq \alpha$. Extension of conditions is defined by: $\left(t_{0}, t_{0}^{*}\right) \leq\left(t_{1}, t_{1}^{*}\right)$ iff $t_{1} \leq t_{0}, t_{1}^{*} \subseteq t_{0}^{*}$ and:

(a) $\left|t_{1}\right| \leq \gamma^{B}<\left|t_{0}\right|, \gamma \in b^{s \uparrow \eta} \in t_{1}^{*} \longrightarrow t_{0}\left(\gamma^{B}\right)=0$ or $s(\eta)$.

(b) $\left|t_{1}\right| \leq \gamma^{C}<\left|t_{0}\right|, \gamma=\left\langle\gamma_{0}, \gamma_{1}\right\rangle, \gamma_{0} \in A \longrightarrow t_{0}\left(\gamma^{C}\right)=0$.

An $R^{s}$-generic is determined by a function $T: \alpha^{+} \longrightarrow 2$ such that $s(\eta)=0$ iff $T\left(\gamma^{B}\right)=0$ for sufficiently large $\gamma \in b^{s \dagger \eta}$ and such that for $\gamma_{0}<\alpha^{+}: \gamma_{0} \in A$ iff $T\left(\gamma^{C}\right)=0$ for sufficiently large $\gamma=\left\langle\gamma_{0}, \gamma_{1}\right\rangle<\alpha^{+}$.

Now we come to the definition of the limit coding, which incorporates the idea of "coding delays". Suppose $s \in S_{\alpha}, \alpha \in \operatorname{Card}^{\prime}$ and $\vec{p}=\left\langle\left(p_{\beta}, p_{\beta}^{*}\right) \mid \beta \in \operatorname{Card} \cap \alpha\right\rangle$ where $p_{\beta} \in S_{\beta}$ for each $\beta \in \operatorname{Card} \cap \alpha$. We wish to define: " $\vec{p}$ codes $s$ ". A natural definition would be: for $\eta<|s|$, 
$p_{\beta}\left(f^{s\lceil\eta}(\beta)\right)=s(\eta)$ for sufficiently large $\beta \in \operatorname{Card} \cap \alpha$. There are problems with this definition however. First, to avoid conflict with the successor coding we should use $f^{s \uparrow \eta}(\beta)^{D}$ instead of $f^{s\lceil\eta}(\beta)$. And it is convenient and sufficient to only require the above for $\beta \in \mathrm{Card}^{+} \cap \alpha$. However, there are still serious difficulties in making sure that the coding of $s$ is consistent with the codings of $p_{\beta}$ by $\vec{p} \uparrow \beta$, for $\beta \in \operatorname{Card}^{\prime} \cap \alpha$. To solve these problems Jensen used $\square$ to make these codings almost disjoint, for singular $\alpha$; this creates new difficulties, resulting from the fact that the singular and inacessible codings are thereby different.

We introduce Coding Delays to facilitate an easier proof of extendibility of conditions. The rough idea is to code $s(\eta)$ not at $f^{s\lceil\eta}(\beta)^{D}$ but instead just after the least ordinal $\geq f^{s \mid \eta}(\beta)^{D}$ where $p_{\beta}$ takes the value 1 .

Definition. Suppose $\alpha \in \operatorname{Card}^{\prime}, s \in S_{\alpha}$. Let $\tilde{\mu}^{s}$ be defined just like $\mu^{s}$ but with the requirement " $\mu^{\prime} \mu=\mu$ for $\mu^{\prime}<\mu$ " replaced by the weaker requirement " $\mu$ a limit ordinal." Then note that $\widetilde{\mathcal{A}}^{s}=L_{\tilde{\mu}^{s}}[A \cap \alpha, s]$ belongs to $\mathcal{A}$, contains $s$ and the $\Sigma_{1}$ Hull $(\alpha \cup\{A \cap \alpha, s\})$ in $\widetilde{\mathcal{A}}^{s}=\widetilde{\mathcal{A}}^{s}$. Now $X$ codes $s$ if $X$ is the $\Sigma_{1}$ theory of $\tilde{\mathcal{A}}^{s}$ with parameters from $\alpha \cup\{A \cap \alpha, s\}$ (viewed as a subset of $\alpha$ ).

Definition. (Limit Coding) Suppose $s \in S_{\alpha}, \alpha \in$ Card $^{\prime}$ and $\vec{p}=\left\langle\left(p_{\beta}, p_{\beta}^{*}\right) \mid \beta \in \operatorname{Card} \cap \alpha\right\rangle$ where $p_{\beta} \in S_{\beta}$ for each $\beta \in \operatorname{Card} \cap \alpha$. We wish to define " $\vec{p}$ codes $s$ ". First we define a sequence $\left\langle s_{\gamma} \mid \gamma \leq \gamma_{0}\right\rangle$ of elements of $S_{\alpha}$ as follows. Let $s_{0}=\phi_{\alpha}$. For limit $\gamma \leq \gamma_{0}$, $s_{\gamma}=\cup\left\{s_{\delta} \mid \delta<\gamma\right\}$. Now suppose $s_{\gamma}$ is defined and let $f_{p}^{s_{\gamma}}(\beta)=$ least $\delta \geq f^{s_{\gamma}}(\beta)$ such that $p_{\beta}\left(\delta^{D}\right)=1$, if such a $\delta$ exists. If $f_{\vec{p}}^{s_{\gamma}}(\beta)$ is undefined for cofinally many $\beta \in \mathrm{Card}^{+} \cap \alpha$ then set $\gamma_{0}=\gamma$. Otherwise define $X \subseteq \alpha$ by: $\delta \in X$ iff $p_{\beta}\left(\left(f_{\vec{p}}^{s_{\gamma}}(\beta)+1+\delta\right)^{D}\right)=1$ for sufficiently large $\beta \in \operatorname{Card}^{+} \cap \alpha$. If Even $(X)$ codes an element $t$ of $S_{\alpha}$ extending $s_{\gamma}$ such that $f_{\vec{p}}^{s_{\gamma}}, X \in \mathcal{A}^{\sqcup}$ then set $s_{\gamma+1}=t$. Otherwise let $s_{\gamma+1}$ be $s_{\gamma} * X^{E}$ if this definition yields $f_{\vec{p}}^{s_{\gamma}} \in \mathcal{A}^{\int_{\gamma+\infty}}$ (and otherwise $\gamma_{0}=\gamma$ ). Now $\vec{p}$ exactly codes $s$ if $s=s_{\gamma}$ for some $\gamma \leq \gamma_{0}$ and $\vec{p}$ codes $s$ if $s \leq s_{\gamma}$ for some $\gamma \leq \gamma_{0}$.

Definition (The Conditions) A condition in $P$ is a sequence $p=\left\langle\left(p_{\alpha}, p_{\alpha}^{*}\right)\right| \alpha \in$ Card, $\alpha \leq$ $\alpha(p))$ where $\alpha(p) \in$ Card and:

(a) $p_{\alpha(p)} \in S_{\alpha(p)}, p_{\alpha(p)}^{*}=\phi$.

(b) For $\alpha \in \operatorname{Card} \cap \alpha(p),\left(p_{\alpha}, p_{\alpha}^{*}\right) \in R^{p_{\alpha}+}$.

(c) For $\alpha \in \operatorname{Card}^{\prime}, \alpha \leq \alpha(p), p\left\lceil\alpha \in \mathcal{A}^{{ }^{\alpha}}, p\left\lceil\alpha\right.\right.$ exactly codes $p_{\alpha}$.

(d) For $\alpha \in \operatorname{Card}^{\prime}, \alpha \leq \alpha(p), \alpha$ inaccessible in $\mathcal{A}^{{ }^{\alpha}}$, there exists CUB $C \subseteq \alpha, C \in \mathcal{A}^{{ }^{\alpha}}$ such that $\beta \in C \longrightarrow p_{\beta}^{*}=\phi$.

Conditions are ordered by: $p \leq q$ iff $\alpha(p) \geq \alpha(q), p(\alpha) \leq q(\alpha)$ in $R^{p_{\alpha}+}$ for $\alpha \in$ Card $\cap \alpha(p) \cap(\alpha(q)+1)$ and $p_{\alpha(p)}$ extends $q_{\alpha(p)}$ if $\alpha(q)=\alpha(p)$.

It is also useful to define some approximations to $P$ : For $\alpha \in \operatorname{Card}, P^{<\alpha}$ denotes the set of all conditions $p$ such that $\alpha(p)<\alpha$. Also for $s \in S_{\alpha}, \omega<\alpha \in \operatorname{Card}, P^{s}$ denotes $P^{<\alpha}$ together with all $p\left\lceil\alpha\right.$ for conditions $p$ such that $\alpha(p)=\alpha, p_{\alpha(p)} \leq s$. To order conditions in $P^{s}$, first define $p^{+}=p$ for $p \in P^{<\alpha}$ and for $p \in P^{s}-P^{<\alpha}, p^{+}\left\lceil\alpha=p\right.$ and $p^{+}(\alpha)=(s\lceil\eta, \phi)$, $\eta$ least such that $p \in P^{s \mid \eta}$; then $p \leq q$ iff $p^{+} \leq q^{+}$as conditions in $P$.

It is worth noting that (c) above implies that $f^{p_{\alpha}}$ dominates the coding of $p_{\alpha}$ by $p \uparrow \alpha$, in the sense that $f^{p_{\alpha}}$ strictly dominates each $f_{p \nmid \alpha}^{p_{\alpha}\lceil\eta}, \eta<\left|p_{\alpha}\right|$ on a tail of $\mathrm{Card}^{+} \cap \alpha$. The purpose of $(\mathrm{d})$ is to guarantee that extendibility of conditions at (local) inaccessibles is not hindered by the Successor Coding (see the proof of Extendibility below). 
We now embark on a series of lemmas which together show that $P$ is the desired forcing: $P$ preserves cofinalities and if $G$ is $P$-generic over $\langle L[A], A\rangle$ then $L[A, G]=L[X]$ for some $X \subseteq \omega_{1}, A$ is $L[X]$-definable from the parameter $X$.

Lemma 5 (Distributivity for $R^{s}$ ) Suppose $\alpha \in$ Card, $s \in S_{\alpha^{+}}$. Then $R^{s}$ is $\alpha^{+}$-distributive in $\mathcal{A}^{\int}$ : if $\left\langle D_{i} \mid i<\alpha\right\rangle \in \mathcal{A}^{\int}$ is a sequence of dense subsets of $R^{s}$ and $p \in R^{s}$ then there is $q \leq p$ such that $q$ meets each $D_{i}$.

Proof Choose $\mu<\mu^{s}$ to be a large enough limit ordinal such that $p,\left\langle D_{i} \mid i<\alpha\right\rangle, \mathcal{A}^{<\int} \in$ $\mathcal{A}=\mathcal{L}_{\mu}\left[\mathcal{A} \cap \alpha^{+}, \int\right]$. Let $\left\langle\alpha_{i} \mid i<\alpha\right\rangle$ enumerate the first $\alpha$ elements of $\left\{\beta<\alpha^{+} \mid \beta=\alpha^{+} \cap \Sigma_{1}\right.$ Hull of $\left(\beta \cup\left\{p,\left\langle D_{i} \mid i<\alpha\right\rangle, \mathcal{A}^{<\int}\right\}\right)$ in $\left.\mathcal{A}\right\}$.

Now write $p$ as $\left(t_{0}, t_{0}^{*}\right)$ and successively extend to $\left(t_{i}, t_{i}^{*}\right)$ for $i \leq \alpha$ as follows: $\left(t_{i+1}, t_{i+1}\right)$ is the least extension of $\left(t_{1}, t_{i}^{*}\right)$ meeting $D_{i}$ such that $t_{i+1}^{*}$ contains $\left\{b^{s \mid \eta}\left|\eta \in H_{i} \cap\right| s \mid\right\}$ where $H_{i}=\Sigma_{1}$ Hull of $\alpha_{i} \cup\left\{p,\left\langle D_{i} \mid i<\alpha\right\rangle, \mathcal{A}^{<\int}\right\}$ in $\mathcal{A}$ and: (a) If $b^{s \uparrow \eta} \in t_{i}^{*}, s(\eta)=1$ then $t_{i+1}\left(\gamma^{\beta}\right)=1$ for some $\gamma \in b^{s \mid \eta}, \gamma>\left|t_{i}\right|$. (b) If $\gamma_{0} \notin A, \gamma_{0}<\left|t_{i}\right|$ then $t_{i+1}\left(\left\langle\gamma_{0}, \gamma_{1}\right\rangle^{C}\right)=1$ for some $\gamma_{1}>\left|t_{i}\right|$.

The lemma reduces to:

Claim $\left(t_{\lambda}, t_{\lambda}^{*}\right)=$ greatest lower bound to $\left\langle\left(t_{i}, t_{i}^{*}\right) \mid i<\lambda\right\rangle$ exists for limit $\lambda \leq \alpha$.

Proof of Claim. We must show that $t_{\lambda}=\cup\left\{t_{i} \mid i<\lambda\right\}$ belongs to $S_{\alpha}$. Note that $\left\langle t_{i} \mid i<\lambda\right\rangle$ is definable over $\bar{H}_{\lambda}=$ transitive collapse of $H_{\lambda}$ and by construction, $t_{\lambda}$ codes $\bar{H}_{\lambda}$ definably over $L_{\bar{\mu}_{\lambda}}\left[t_{\lambda}\right]$, where $\bar{\mu}_{\lambda}=$ height of $\bar{H}_{\lambda}$. So $t_{\lambda}$ is reshaped, as $\left|t_{\lambda}\right|$ is singular, definably over $L_{\bar{\mu}_{\lambda}}\left[t_{\lambda}\right]$. By Lemma $1, \bar{\mu}_{\lambda}<\left(\left|t_{\lambda}\right|^{+}\right)^{L}$ if $\alpha \geq \omega_{2}$. So $t_{\lambda}$ belongs to $S_{\alpha} \cdot \dashv$

The next lemma illustrates the use of coding delays:

Lemma 6 (Extendibility for $P^{s}$ ) Suppose $p \in P^{s}, s \in S_{\alpha}, X \subseteq \alpha, X \in \mathcal{A}^{\int}$. Then there exists $q \leq p$ such that $X \cap \beta \in \mathcal{A}^{\amalg_{\beta}}$ for each $\beta \in \operatorname{Card} \cap \alpha$.

Proof Let $Y \subseteq \alpha$ be chosen so that Even $(Y)$ codes $s$ and Odd $(Y)$ is the $\Sigma_{1}$ theory of $\mathcal{A}$ with parameters from $\alpha \cup\{A \cap \alpha, s\}$, where $\mathcal{A}$ is an initial segment of $\mathcal{A}^{\mathcal{J}}$ large enough to extend $\widetilde{\mathcal{A}}^{f}$ and to contain $X, p$. For $\beta \in \operatorname{Card} \cap \alpha$, let $\overline{\mathcal{A}}_{\beta}=$ transitive collapse of $\Sigma_{1}$ Hull $(\beta \cup\{A \cap \alpha, s\})$ in $\mathcal{A}$, and $g(\beta)=\beta^{+}$of $\overline{\mathcal{A}}_{\beta}$.

Define $q$ as follows: $q_{\beta}=s_{\beta}$ if Even $(Y \cap \beta)$ codes $s_{\beta} \in S_{\beta}, q_{\beta}=p_{\beta} *(Y \cap \beta)^{E}$ for other $\beta \in \operatorname{Card}^{\prime} \cap \alpha, q_{\beta}=p_{\beta} * \vec{O} * 1 *(Y \cap \beta)^{D}$ where $\vec{O}$ has length $g(\beta)$ for $\beta \in \operatorname{Card}^{+} \cap \alpha$. And $q_{\beta}^{*}=p_{\beta}^{*}$ for all $\beta \in$ Card $\cap \alpha$.

As $g\left\lceil\beta, Y \cap \beta\right.$ are definable over $\overline{\mathcal{A}}_{\beta}$ for $\beta \in \operatorname{Card} \cap \alpha$ we get $g \uparrow \beta, Y \cap \beta \in \mathcal{A}^{\int_{\beta}}$ when $\operatorname{Even}(Y \cap \beta)$ codes $s_{\beta} \in S_{\beta}$. Also $g\left\lceil\beta, Y \cap \beta \in \mathcal{A}^{\amalg_{\beta}}\right.$ for other $\beta \in \operatorname{Card}^{\prime} \cap \alpha$ as $\operatorname{Odd}(Y \cap \beta)$ codes $\overline{\mathcal{A}}_{\beta}$. And note that for all $\beta \in \operatorname{Card}^{\prime} \cap \alpha, g\left\lceil\beta\right.$ dominates $f^{p_{\beta}}$ on a final segment of $\operatorname{Card}^{+} \cap \beta$, unless Even $(Y \cap \beta)$ codes $s_{\beta}=p_{\beta}$, in which case $q\left\lceil\beta\right.$ exactly codes $s_{\beta}$ because $p\lceil\beta$ does.

So we conclude that $q \uparrow \beta$ exactly codes $q_{\beta}$ for sufficiently large $\beta \in \operatorname{Card}^{\prime} \cap \alpha$ and clearly $X \cap \beta \in \mathcal{A}^{\amalg_{\beta}}$ for such $\beta$. Apply induction on $\alpha$ to obtain this for all $\beta \in \operatorname{Card}^{\prime} \cap \alpha$. Finally, note that the only problem in verifying $q \leq p$ is that the restraint $p_{\beta}^{*}$ may prevent us from making the extension $q_{\beta}$ of $p_{\beta}$ when $q_{\beta}=s_{\beta}$, Even $(Y \cap \beta)$ codes $s_{\beta}$. But property (d) in the definition of condition guarantees that $p_{\beta}^{*}=\phi$ for $\beta$ in a CUB $C \subseteq \alpha, C \in \mathcal{A}^{\int}$. We may assume that $C \in \mathcal{A}$ and hence for sufficiently large $\beta$ as above we get $\beta \in C$ and hence 
$p_{\beta}^{*}=\phi$. So $q \leq p$ on a final segment of $\operatorname{Card} \cap \alpha$, and we may again apply induction to get $q \leq p$ everywhere. $\dashv$

The key idea of Jensen's proof lies in the verification of distributivity for $P^{s}$. Before we can state and prove this property we need some definitions.

Definition Suppose $\beta \in \operatorname{Card}^{+} \cap \alpha$ and $D \subseteq P^{s}, s \in S_{\alpha}$. D is $\beta$-dense on $P^{s}$ if $\forall p \in P^{s} \exists q \in$ $P^{s}\left(q \leq p, q\right.$ meets $D$ and $q \uparrow \beta=p\lceil\beta)$. $X \subseteq$ Card $\cap \alpha$ is thin in $\mathcal{A}^{\int}$ if $X \in \mathcal{A}^{\int}$ and for each inaccessible $\beta \leq \alpha, \mathcal{A}^{\mathcal{J}} \models \mathcal{X} \cap \beta$ is not stationary in $\beta$. A function $f: \operatorname{Card} \cap \alpha \longrightarrow V$ in $\mathcal{A}^{\int}$ is small in $\mathcal{A}^{\int}$ if for each $\beta \in \operatorname{Card} \cap \alpha, f(\beta) \in H_{\beta^{++}}^{\mathcal{A}^{\jmath}}, \operatorname{card}(f(\beta)) \leq \beta$ in $\mathcal{A}$ and Support $(f)=\{\beta \in \operatorname{Card} \cap \alpha \mid f(\beta) \neq \phi\}$ is thin in $\mathcal{A}^{\int}$. If $D \subseteq P^{s}$ is predense and $p \in P^{s}, \beta \in$ Card we say that $p$ reduces $D$ below $\beta$ if for some $\gamma \in \operatorname{Card}^{+} \gamma \leq \beta,\{r \mid r \cup p\lceil[\gamma, \alpha)$ meets $D\}$ is predense on $P^{p_{\gamma}}$ below $p\left\lceil\gamma\right.$. Finally, for $p \in P^{s}, f$ small in $\mathcal{A}^{\int}$ we define $\Sigma_{f}^{p}=$ all $q \leq p$ in $P^{s}$ such that whenever $\beta \in \operatorname{Card} \cap \alpha, D \in f(\beta), D$ predense on $P^{p^{+}}$then $q$ reduces $D$ below $\beta$.

Lemma 7 (Distributivity for $P^{s}$ ) Suppose $s \in S_{\beta^{+}}, \beta \in$ Card .

(a) If $\left\langle D_{i} \mid i<\beta\right\rangle \in \mathcal{A}^{\int} ; \mathcal{D}_{\rangle} i^{+}$-dense on $P^{s}$ for each $i<\beta$ and $p \in P^{s}$ then there is $q \leq p$, $q$ meets each $D_{i}$.

(b) If $p \in P^{s}, f$ small in $\mathcal{A}^{\int}$ then there exists $q \leq p, q \in \Sigma_{f}^{p}$.

Proof We demonstrate (a) and (b) by a simultaneous induction on $\beta$. If $\beta=\omega$ or belongs to $\mathrm{Card}^{+}$then by induction (a) reduces to the $\beta^{+}$-distributivity of $R^{s}$ in $\mathcal{A}^{\int}$, Lemma 5 . And (b) reduces to: if $S$ is a collection of $\beta$-many predense subsets of $P^{s}, S \in \mathcal{A}^{\int}$ then $\left\{q \in P^{s} \mid q\right.$ reduces each $D \in S$ below $\left.\beta\right\}$ is dense on $P^{s}$. Again this follows from Lemma 5 since $P^{s}$ factors as $R^{s} * Q$ where $1^{R^{s}} \Vdash Q$ is $\beta^{+}-c c$, and hence any $p \in P^{s}$ can be extended to $q \in P^{s}$ such that $D^{q}=\left\{r \in D \mid q(\beta) \leq r(\beta)\right.$ in $\left.R^{s}\right\}$ is predense $\leq q$ for each $D \in S$ and hence $q$ reduces each $D \in S$ below $\beta$.

Now suppose that $\beta$ is inaccessible. We first show that (b) holds for $f$, provided $f(\beta)=\phi$. First select a CUB $C \subseteq \beta$ in $\mathcal{A}^{f}$ such that $\gamma \in C \longrightarrow f(\gamma)=\phi$ and extend $p$ so that $f \uparrow \gamma, C \cap \gamma$ belong to $\mathcal{A} \sqrt{ }^{\gamma}$ for each $\gamma \in \operatorname{Card} \cap \beta^{+}$. Then we can successively extend $p$ on $\left[\beta_{i}^{+}, \beta_{i+1}\right]$ in the least way so as to meet $\Sigma_{f}^{p}$ on $\left[\beta_{i}^{+}, \beta_{i+1}\right]$, where $\left\langle\beta_{i} \mid i<\beta\right\rangle$ is the increasing enumeration of $\mathrm{C}$. At limit stages $\lambda$, we still have a condition, as the sequence of first $\lambda$ extensions belongs to $\mathcal{A} \sqrt{ }^{\beta_{\lambda}}$. The final condition, after $\beta$ steps, is an extension of $p$ in $\Sigma_{f}^{p}$.

Now we prove (a) in this case. Suppose $p \in P^{s}$ and $\left\langle D_{i} \mid i<\beta\right\rangle \in \mathcal{A}^{\int}, D_{i}$ is $i^{+}$-dense on $P^{s}$ for each $i<\beta$. Let $\mu_{0}<\mu^{s}$ be a big enough limit ordinal so that $\left\langle D_{i} \mid i<\beta\right\rangle, p, \tilde{\mu}^{s} \in L_{\mu_{0}}$ $\left[A \cap \beta^{+}, s\right]$ and for $i<\beta$ let $\mu_{i}=\mu_{0}+\omega \cdot i<\mu^{s}$. For any $X$ we let $H_{i}(X)$ denote $\Sigma_{1}$ $\operatorname{Hull}\left(X \cup\left\{\left\langle D_{i} \mid i<\beta\right\rangle, p, \tilde{\mu}^{s}, s, A \cap \beta^{+}\right\}\right)$in $L_{\mu_{i}}\left[A \cap \beta^{+}, s\right]$.

Let $f_{i}: \operatorname{Card} \cap \beta \longrightarrow V$ be defined by: $f_{i}(\gamma)=H_{\gamma^{+}} \cap H_{i}(\gamma)$ if $i<\gamma \in H_{i}(\gamma)$, $i<\gamma<\beta$ and $f_{i}(\gamma)=\phi$ otherwise. Then each $f_{i}$ is small in $\mathcal{A}^{\int}$ and we inductively define $p=p^{0} \geq p^{1} \geq \ldots$ in $P^{s}$ as follows: $p^{i+1}=$ least $q \leq p^{i}$ such that:

(a) $q(\beta)$ meets all predense $D \subseteq R^{s}, D \in H_{i}(\beta)$.

(b) $q$ meets $\Sigma_{f_{i}}^{p^{i}}$ and $D_{i}$.

(c) $q\left\lceil i^{+}=p^{i}\left\lceil i^{+}\right.\right.$.

For limit $\lambda \leq \beta$ we take $p^{\lambda}$ to be the greatest lower bound to $\left\langle p^{i} \mid i<\lambda\right\rangle$, if it exists. 
Claim $p^{\lambda}$ is a condition in $P^{s}$, where $p^{\lambda}(\gamma)=\left(\cup\left\{p_{\gamma}^{i} \mid i<\lambda\right\}, \cup\left\{p_{\gamma}^{i *} \mid i<\lambda\right\}\right)$ for each $\gamma \in \operatorname{Card} \cap \beta^{+}$.

First we verify that $p_{\gamma}^{\lambda}=\cup\left\{p_{\gamma}^{i} \mid i<\lambda\right\}$ belongs to $S_{\gamma}$. Let $\bar{H}_{\lambda}(\gamma)$ be the transitive collapse of $H_{\lambda}(\gamma)$ and write $\bar{H}_{\lambda}(\gamma)$ as $L_{\bar{\mu}}[\bar{A}, \bar{s}], \bar{P}=$ image of $P^{s} \cap H_{\lambda}(\gamma)$ under transitive collapse, $\bar{\beta}=$ image of $\beta$ under collapse. Also write $\bar{P}$ as $R^{\bar{s}} * P^{\bar{G}_{\bar{\beta}}}$ where $\bar{G}$ denotes an $R^{\bar{s}}$-generic (just as $P^{s}$ factors as $R^{s} * P^{G_{\beta}}, G_{\beta}$ denoting an $R^{s}$-generic).

Now the construction of the $p^{i}$ 's (see conditions (a), (b)) was designed to guarantee that if $\gamma \in H_{\lambda}(\gamma)$ then $\bar{G}_{\bar{\beta}}=\left\{\bar{p} \in R^{\bar{s}} \mid \bar{p}\right.$ is extended by some $\left.\bar{p}^{i}(\bar{\beta})\right\}$ is $R^{\bar{s}}$-generic over $\bar{H}_{\lambda}(\gamma)$, where $\bar{p}^{i}=$ image of $p^{i}$ under collapse, and that for each $\gamma<\bar{\delta}<\bar{\beta}$ in $\operatorname{Card}^{+}\left(\bar{H}_{\lambda}(\gamma)\right),\{\bar{p} \mid \bar{p}$ is extended by some $\bar{p}^{i} \uparrow[\gamma, \bar{\delta})$ in $\left.\bar{P}_{\gamma}^{\bar{p}_{\bar{\delta}}^{i}}\right\}$ is $\bar{P}_{\gamma}^{\bar{G}_{\bar{\delta}}}$-generic over $\mathcal{A}^{<\overline{\mathcal{G}}_{\bar{\delta}}}=\cup\left\{\mathcal{A}^{<\sqrt{\delta}^{\bar{\delta}}}|\rangle<\lambda\right\}$ where $\bar{P}_{\gamma}^{\bar{p}_{\bar{\delta}}^{i}}$ denotes the image under collapse of $P_{\gamma}^{p_{\delta}^{i}}=\left\{q \uparrow[\gamma, \delta) \mid q \in P^{p_{\delta}^{i}}\right\}, \bar{\delta}=$ image of $\delta$ under collapse.

Note: We do not necessarily have the previous claim for $\bar{\delta}=\bar{\beta}$, and this is the source of our need for $\sim O^{\#}$ in this proof.

By induction, we have the distributivity of $P^{t}$ for $t \in S_{\delta}, \delta \in \operatorname{Card}^{+} \cap \beta$, and hence that of $\bar{P}^{\bar{t}}$ for $\bar{t} \in \bar{S}_{\bar{\delta}}, \bar{\delta} \in \operatorname{Card}^{+}\left(\bar{H}_{\lambda}(\gamma)\right), \bar{\delta}<\bar{\beta}$. So the "weak" genericity of the preceding paragraph implies that:

(d) $L_{\bar{\beta}}\left[A \cap \gamma, p_{\gamma}^{\lambda}\right] \models p_{\gamma}^{\lambda} \mid$ is a cardinal. Also:

(e) $L_{\bar{\mu}}\left[A \cap \gamma, p_{\gamma}^{\lambda}\right] \models\left|p_{\gamma}^{\lambda}\right|$ is $\Sigma_{1}$-singular.

Thus $p_{\gamma}^{\lambda} \in S_{\gamma}$ (by (e)) provided we can show that when $\gamma \geq \omega_{2}, \bar{\mu}<\left(\left|p_{\gamma}^{\lambda}\right|^{+}\right)^{L}$. But $\bar{H}_{\lambda}(\gamma) \stackrel{\sim}{\longrightarrow} H_{\lambda}(\gamma)$ gives a $\Sigma_{1}$-elementary embedding with critical point $\left|p_{\gamma}^{\lambda}\right|$, so by Lemma 1 , this is true. Also note that we now get $p^{\lambda} \uparrow \gamma \in \mathcal{A}^{\sqrt{\gamma}^{\lambda}}$ as well, since $p^{\lambda} \uparrow \gamma$ is definable over $\bar{H}_{\lambda}(\gamma)$ and we defined $\mathcal{A}^{\sqrt{\gamma}^{\gamma}}$ to be large enough to contain $\bar{H}_{\lambda}(\gamma)$, since $L_{\bar{\beta}} \models\left|p_{\gamma}^{\lambda}\right|$ is a cardinal by $(d)$.

The previous argument applies also if $\gamma=\beta$, using the distributivity of $R^{s}$, or if $\gamma=$ $\beta \cap H_{\lambda}(\gamma)$, using the fact that $p_{\beta}^{\lambda}$ collapses to $p_{\gamma}^{\lambda}$. If $\gamma<\gamma^{*}=\min \left(H_{\lambda}(\gamma) \cap[\gamma, \beta)\right)$ then we can apply the first argument to get the result for $\gamma^{*}$, and then the second argument to get the result for $\gamma$.

Finally, to prove the Claim we must verify the restraint condition (d) in the definition of $P$. Suppose $\gamma$ is inaccessible and for $i<\lambda$ let $C^{i}$ be the least CUB subset of $\gamma$ in $\mathcal{A}^{\gamma^{\gamma}}$ disjoint from $\left\{\bar{\gamma}<\gamma \mid p_{\bar{\gamma}}^{i} * \neq\right\}$. If $\lambda<\gamma$ then $\bigcap\left\{C^{i} \mid i<\lambda\right\}$ witnesses the restraint condition for $p^{\lambda}$ at $\gamma$, if $\gamma<\lambda$ then the restraint condition for $p^{\lambda}$ at $\gamma$ follows by induction on $\lambda$ and if $\gamma=\lambda$ then $\Delta\left\{C^{i} \mid i<\lambda\right\}$ witnesses the restraint condition for $p^{\lambda}$ at $\gamma$, where $\Delta$ denotes diagonal intersection.

Thus the Claim and therefore (a) is proved in case $\beta$ is inaccessible. To verify (b) in this case, note that as we have already proved (b) when $f(\beta)=\phi$ it suffices to show: if $\left\langle D_{i} \mid i<\beta\right\rangle \in \mathcal{A}^{\int}$ is a sequence of dense subsets of $P^{s}$ then $\forall p \exists q \leq p$ ( $q$ reduces each $D_{i}$ below $\beta$ ). But using distributivity we see that $D_{i}^{*}=\left\{q \mid q\right.$ reduces $D_{i}$ below $\left.i^{+}\right\}$is $i^{+}$-dense for each $i<\beta$ so again by distributivity there is $q \leq p$ reducing each $D_{i}$ below $i^{+}$.

We are now left with the case where $\beta$ is singular. The proof of (a) can be handled using the ideas from the inaccessible case, as follows. Choose $\left\langle\beta_{i} \mid i<\lambda_{0}\right\rangle$ to be a continuous and 
cofinal sequence of cardinals $<\beta, \lambda_{0}<\beta_{0}$. First, we argue that $p \in P^{s}$ can be extended to meet $\Sigma_{f}^{p}$ for any $f$ small in $\mathcal{A}^{\mathcal{f}}$, provided $f(\beta)=\phi$ : Extend $p$ if necessary so that for each $\gamma \in \operatorname{Card} \cap \beta^{+}, f \uparrow \gamma$ and $\left\{\beta_{i} \mid \beta_{i}<\gamma\right\}$ belong to $\mathcal{A}{ }^{\gamma}$. Now perform a construction like the one used to prove distributivity in the inacessible case, extending $p$ successively on $\left[\beta_{0}, \beta_{i}^{+}\right]$ so as to meet $\Sigma_{f}^{p}$ on $\left[\beta_{0}, \beta_{i}^{+}\right]$as well as appropriate $\Sigma_{f_{i}}^{p_{i}}$ s defined on $\left[\beta_{0}, \beta_{i}^{+}\right]$to guarantee that $p^{\lambda}$ is a condition for limit $\lambda \leq \lambda_{0}$. Note that each extension is made on a bounded initial segment of $\left[\beta_{0}, \beta\right)$ and therefore by induction $\Sigma_{f}^{p}, \Sigma_{f_{i}}^{p_{i}}$ can be met on these intervals. The result is that $p$ can be extended to meet $\Sigma_{f}^{p}$ on a final segment of Card $\cap \beta$ and therefore by induction can be extended to meet $\Sigma_{f}^{p}$. Second, use the density of $\Sigma_{f}^{p}$ when $f(\beta)=\phi$ to carry out the distributivity proof as we did in the inaccessible case. And again, (b) follows from (a). This completes the proof of Lemma $7 . \dashv$

Now the same argument as used above also shows:

Lemma 8 (Distributivity for $P$ ) If $\left\langle D_{i} \mid i<\beta\right\rangle$ is $\langle L[A], A\rangle$-definable, each $D_{i}$ is $i^{+}$-dense on $P$ and $p \in P$ then there exists $q \leq p, q$ meets each $D_{i}$.

Extendibility for $P^{s}$ and Distributivity for $P$ give us the conclusions of Theorem 4 . This completes the proof.

\section{References}

Beller-Jensen-Welch [82] Coding the Universe, Cambridge University Press.

Friedman [94] A Simpler Proof of Jensen's Coding Theorem, Annals of Pure and Applied Logic, vol. 70, No.1, pages 1-16.

Jensen-Solovay [68] Some Applications of Almost Disjoint Sets, in Mathematical Logic and the Foundations of Set Theory, North Holland, pages 84-104. 